\title{
Marcapasos diafragmático en paciente pediátrico con síndrome de Hipoventilación Central Adquirido
}

\author{
Diaphragm pacing in a pediatric patient with Acquired Central \\ Hypoventilation syndrome
}

\section{Javier Varela O. ${ }^{a}$, Germán Sepúlveda I. ${ }^{a}$, Jose Sepúlveda C. ${ }^{b}$, Renato Acuña L. ${ }^{c}$, Gustavo Pizarro T. ${ }^{\text {b , Viviana Maldonado U. }}{ }^{\text {bdd }}$, Alejandra Alvarado G. ${ }^{\text {b,d }}$}

\author{
a Unidad Paciente Crítico Pediátrico, Hospital Clínico de Magallanes, Universidad de Magallanes. Chile \\ ${ }^{b}$ Centro de Responsabilidad Infantil, Hospital Clínico de Magallanes. Chile \\ cHospital Padre Hurtado. Clínica Alemana de Santiago. Chile \\ dKinesióloga
}

Recibido: 18 de marzo de 2019; Aceptado: 18 de septiembre de 2019

\section{¿Qué se sabe del tema que trata este estudio?}

El marcapasos diafragmático permite disminuir o eliminar la necesidad de ventilación mecánica en pacientes con hipoventilación central. A la fecha la mayor parte de los casos publicados están relacionados con lesión medular alta y síndrome de hipoventilación central congénito.
¿Qué aporta este estudio a lo ya conocido?

Este documento aporta una descripción detallada sobre la implantación de un marcapasos diafragmático en paciente pediátrico con síndrome de hipoventilación central adquirido y apoya el hecho de que en este grupo de pacientes es una opción factible y potencialmente segura.

\section{Resumen}

El marcapasos diafragmático permite reducir o eliminar la necesidad de ventilación mecánica en pacientes con insuficiencia respiratoria crónica que conservan el eje nervio frénico-diafragma intacto, siempre que no presenten enfermedad pulmonar intrínseca. Aunque su implantación ha sido practicada por décadas, su uso no está ampliamente difundido, y existe poca literatura publicada al respecto, la mayoría relacionada con lesión medular alta y síndrome de hipoventilación central congénito. Objetivo: Describir una experiencia de implantación de marcapasos diafragmático en paciente pediátrico con síndrome de hipoventilación central adquirido. Caso Clínico: $\mathrm{Pa}$ ciente femenino con síndrome de hipoventilación central secundario a lesión isquémica de tronco cerebral como resultado de disfunción de válvula de derivación ventrículo peritoneal, motivo por el cual durante 5 años se mantuvo con asistencia de ventilación mecánica intrahospitalaria. A los 7 años de edad se implantó marcapasos diafragmático mediante cirugía toracoscópica, lo que permitió posterior a un periodo de rehabilitación y acondicionamiento respiratorio el destete de la ventilación mecánica y el egreso hospitalario. Conclusiones: El marcapasos diafragmático es una opción factible, potencialmente segura y costo efectiva para disminuir o eliminar la dependencia de ventilación mecánica y mejorar la calidad de vida en pacientes con síndrome de hipoventilación central adquirido.
Palabras clave:

Marcapasos diafragmático; cirugía toracoscópica; síndrome hipoventilación central; insuficiencia respiratoria 


\begin{abstract}
Diaphragmatic pacemaker is a device that reduces or eliminates the need of mechanical ventilation in patients with chronic respiratory failure who keep the phrenic nerve-diaphragm axis intact, as long as they do not present intrinsic lung disease. Although its implantation has been practiced for decades, its use is not widespread and to date, there is little published literature about it, mostly related to high spinal cord injury and congenital central hypoventilation syndrome. Objective: To describe an experience of diaphragmatic pacemaker implantation in a pediatric patient with acquired central hypoventilation syndrome. Clinical Case: Female patient with central hypoventilation syndrome secondary to ischemic brainstem lesion as a result of ventriculoperitoneal shunt malfunction. For this reason, for 5 years she was supported by inpatient mechanical ventilation. At 7 years of age, a diaphragmatic pacemaker was implanted by thoracoscopic surgery, which allowed, after a period of rehabilitation and respiratory conditioning, mechanical ventilation withdrawal, and hospital discharge. Conclusions: Diaphragmatic pacemaker is a feasible, potentially safe, and cost-effective option for decreasing or eliminating mechanical ventilation dependence and improve life quality in patients with acquired central hypoventilation syndrome.
\end{abstract}

\section{Keywords:}

Diaphragm pacing; thoracoscopic surgery; central hypoventilation syndrome; respiratory insufficiency

\section{Introducción}

El síndrome de hipoventilación central (SHC) es una condición poco frecuente que conlleva a insuficiencia respiratoria crónica. Se caracteriza por una desconexión entre los centros respiratorios del tronco cerebral y el nervio frénico, lo que genera alteración en la regulación autonómica y respiratoria. Este puede ser idiopático como en el caso del SHC congénito (enfermedad de Ondine) o adquirido como resultado de infartos en el tronco cerebral, tumores, trauma quirúrgico, hemorragia o meningoencefalitis. El manejo de estos pacientes requiere el uso de ventilación mecánica (VM), en algunos casos por $24 \mathrm{~h}$ al día y en otros exclusivamente durante el sueño ${ }^{1,2}$.

En 1966 Glenn fue el primero en describir el uso del marcapasos diafragmático (MD) y posteriormente Hunt desarrolló un método modificado para uso en niños, permitiendo actualmente implantarse a edades tan tempranas como 9 meses $^{1,3-5}$. Este dispositivo provee de una corriente eléctrica al nervio frénico generando una contracción diafragmática. Consta de un emisor de radiofrecuencia, una antena, un receptor y un electrodo ${ }^{6}$ (figura 1 y 2 ). La estimulación del nervio frénico permite eliminar o disminuir la necesidad de $\mathrm{VM}$ en pacientes con insuficiencia respiratoria crónica que conservan el eje nervio frénico-diafragma intacto, siempre que no presenten enfermedad pulmonar intrínseca u obesidad ${ }^{1,5,7,8}$

El electrodo del MD puede implantarse a través del cuello o el tórax, cuando se emplea la vía torácica la cirugía puede ser abierta o mediante toracoscopia ${ }^{1}$. La técnica toracoscópica es relativamente infrecuente. En 1998 Shaul documentó por primera vez la utilización de este método ${ }^{9} \mathrm{y}$ posteriormente el mismo autor reporta una serie de 9 pacientes en la que se refieren complicaciones como atelectasia, neumonía, neumotórax, bradicardia y necesidad de reinstalación ${ }^{10}$. Recientemente Nicholson reporta una serie de 18 niños con SHC congénito, encontrando un menor índice de complicaciones ${ }^{5}$.

Aunque la implantación del MD ha sido practicada por varias décadas, su uso no está ampliamente difundido y existe todavía mucho trabajo en innovación ${ }^{8}$. A la fecha hay poca literatura publicada y la mayoría está relacionada con lesión medular alta y SHC congénito. El objetivo de la presente publicación es describir una experiencia exitosa de implantación de MD en paciente pediátrico con SHC adquirido.

\section{Caso Clínico}

Paciente del género femenino, de actualmente 9 años de edad, con hidrocefalia congénita e hipoplasia del cuerpo calloso a quien se instaló válvula de derivación ventrículo peritoneal en el periodo neonatal. A los 2 años de edad ingresó a nuestro hospital con signos clínicos de enclavamiento secundario a disfunción valvular. Posterior a este evento evolucionó con pérdida del control automático de la respiración con dependencia de VM y alteración de la mecánica de la deglución, motivo por el cual se realizó traqueostomía y gastrostomía. En la evolución subsecuente se encontró dismetría y compromiso parcial de pares craneanos $\mathrm{V}$ al XII, retraso del desarrollo psicomotor leve y epilepsia.

En resonancia magnética cerebral destacaba atrofia de ambos hemisferios cerebelosos, del vermis superior y del tronco cerebral, principalmente en región dorsal del bulbo raquídeo. Se manejó durante 5 años con VM intrahospitalaria tolerando ventanas en filtro 
de duración variable ( 3 a 6 h) durante el estado de vigilia, situación durante la cual mantenía el control voluntario de la respiración, lo que permitió evaluar de forma clínica la integridad del eje nervio frénicodiafragma.

A los 7 años de edad se instaló MD mediante toracoscopia y bajo anestesia general con técnica de "single lung ventilation". Se accedió a ambos lados del tórax en diferentes tiempos. Se disecó el nervio frénico en la zona adyacente al hilio pulmonar cuando se intervino el lado derecho (entre t5 y t6) y sobre el borde superior del pericardio cuando se trabajó el nervio frénico izquierdo. Luego se introdujo el electrodo al tórax en la zona donde finalmente se alojó también el receptor y se pasó debajo de cada nervio fijándose a los tejidos circundantes. Posteriormente se creó un túnel para el cable del electrodo en el tejido subcutáneo, un bolsillo para alojar al receptor (figura 3) y se probó la correcta estimulación. No hubo complicaciones intraoperatorias ni postoperatorias.

Previo a la intervención quirúrgica se llevó a cabo durante 3 meses programa de rehabilitación respiratoria que incluyó: kinesioterapia respiratoria tradicional, entrenamiento de la musculatura respiratoria y entrenamiento cardiopulmonar. El entrenamiento de la musculatura respiratoria se llevó a cabo con válvula de entrenamiento muscular inspiratorio adaptada a la cánula de traqueostomía. Una vez evaluada la presión inspiratoria máxima, se realizó entrenamiento con $30 \%$ de carga inspiratoria 15 min diarios alternando 3 min de trabajo por 2 min de descanso, durante 5 días a la semana. Paralelamente se realizó entrenamiento cardiopulmonar con bicicleta estática, 2 veces a la semana durante $30 \mathrm{~min}$ al día, alternando intervalos de 10 min de trabajo por 5 min de descanso.

Posterior a la instalación del MD, se mantuvo durante 30 días con VM exclusiva. La programación del marcapasos se realizó guiada por visualización de expansión torácica, ventilometría, gases arteriales, capnografía y SpO2. En primer lugar, se estableció la amplitud de trabajo para cada hemidiafragma entre el umbral inferior, correspondiente al estímulo mínimo que generaba expansión torácica y el umbral superior, correspondiente al estímulo que no generaba gran cambio en la expansión. Posteriormente se ajustó la amplitud entre estos valores estableciendo como objetivo el volumen corriente que movilizaba en VM. Para programar la frecuencia respiratoria se usó de forma inicial la que manejaba en VM y luego se ajustó de acuerdo a gases arteriales y capnografía.

Paralelamente, se estableció protocolo para destete de VM, iniciando el uso del dispositivo durante 1 hora al día la primera semana y $2 \mathrm{~h}$ al día la segunda semana. Posteriormente se incrementó en rangos de $2 \mathrm{~h}$ por

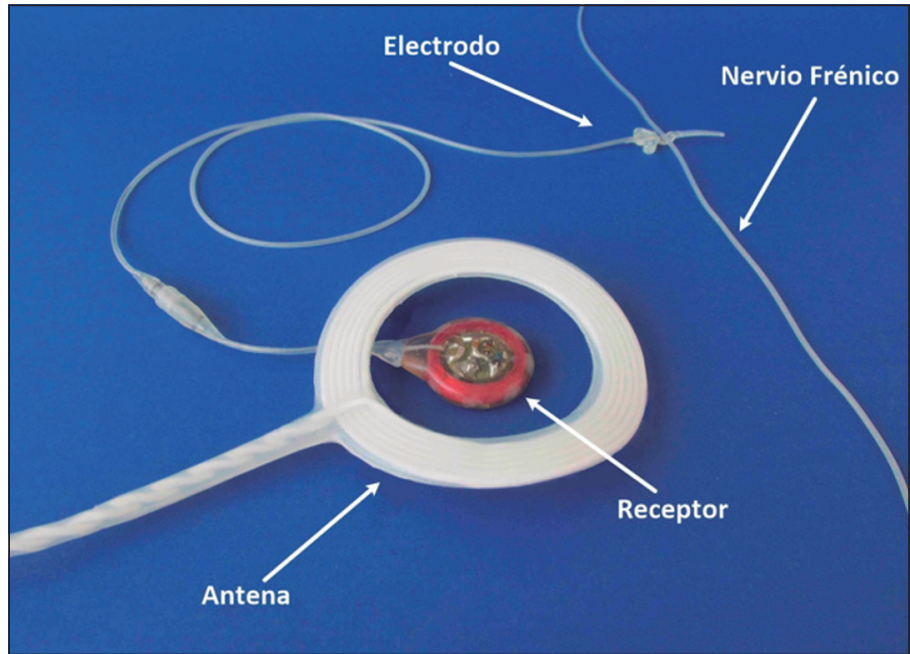

Figura 1. Componentes del marcapasos diafragmático y esquematización de contacto del electrodo con el nervio frénico.

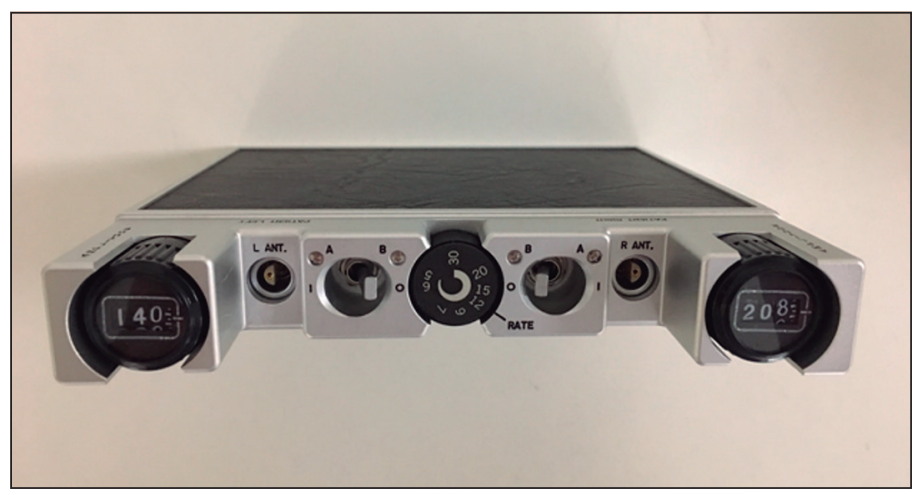

Figura 2. Emisor de radiofrecuencia.

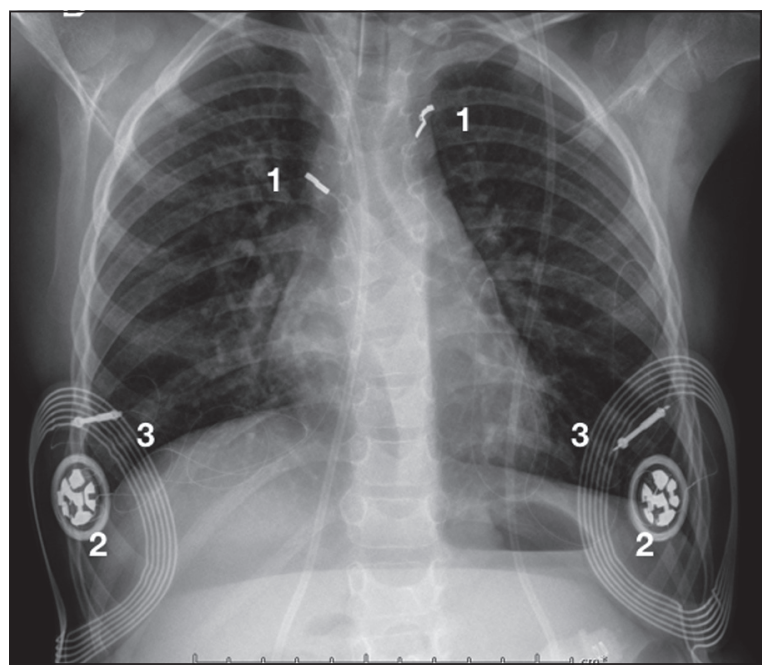

Figura 3. Radiografía de tórax postero-anterior. Se observa: (1) Electrodo en contacto con nervio frénico bilateral. (2) Receptor alojado subcutáneo a ambos lados. (3) Antena de marcapasos adosada a la piel a ambos lados. 
semana hasta completar $14 \mathrm{~h}$ diurnas, dicho objetivo se alcanzó a las 16 semanas postquirúrgicas y se mantuvo así durante 6 semanas. Posterior a este periodo se suspendió el uso de VM nocturna quedando solo asistida por el marcapasos.

A 2 años de seguimiento, la paciente fue egresada del hospital, manteniéndose con cánula de traqueostomía, válvula de fonación y sin complicaciones en su evolución.

\section{Discusión}

En el presente reporte destacamos que la implantación de un MD permitió que un paciente pediátrico con SHC adquirido fuera destetado de VM y egresado del hospital, lo que ha resultado en mejoría de su calidad de vida, disminución en infecciones asociadas a VM y en los costos de atención.

Uno de los objetivos principales de la colocación del MD es mejorar la calidad de vida. Romero-Ganuza aplicó cuestionarios para valorar calidad de vida en pacientes con lesión medular, encontrando que aquellos a los que se les colocó MD comparado con los que permanecieron en VM, perciben mejor calidad de vida de forma significativa en los ámbitos de seguridad, comunicación, sociabilidad, comodidad y movilidad. El mismo estudio reporta que el $78,4 \%$ de los pacientes a los que se les colocó MD tienen como destino de egreso el domicilio familiar a diferencia del $51,6 \%$ del grupo con $\mathrm{VM}^{4}$. En nuestro caso dado la edad de la paciente no se aplicaron instrumentos para evaluar la calidad de vida; sin embargo, después de 5 años de estancia en el hospital pudo ser egresada e integrada a la vida familiar, sumado a la mejoría en su movilización una vez destetado de VM, suponen una mejor calidad de vida.

El MD permite disminuir la incidencia de infecciones respiratorias asociadas a VM, tal como se demuestra en el estudio de Hirschfeld, en el que se encontró que en los pacientes con MD la frecuencia de infecciones del tracto respiratorio disminuye de forma significativa $^{12}$. La paciente del presente reporte posterior a la implantación del dispositivo y el alta hospitalaria se ha mantenido libre de infecciones.

Varios estudios sugieren que la utilización del MD reduce costos a largo plazo ${ }^{1,2,12}$. Hirschfeld encuentra que la disminución de infecciones respiratorias y la menor necesidad de recursos humanos y tecnológicos comparado con VM reduce los costos de forma significativa $^{12}$. Hasta el momento es difícil decir que en nuestro caso la instalación del MD disminuyó los costos; sin embargo, el hecho que se haya egresado del hospital supone en adelante una disminución significativa.
Romero reporta un tiempo promedio para destetar de la VM después de la implantación del MD de 47,33 días (31-96) $)^{11}$ e Hirschfeld de 51,06 días $(30-196)^{12}$. Ambos estudios realizados en pacientes con cuadriplejia secundario a lesión medular alta. En el estudio de Nicholson realizado en pacientes con SHC congénito el promedio fue de 5,3 meses (159 días) con intervalo de 2,7 a 9,7 meses $^{5}$. En nuestra paciente el tiempo fue de 154 días, muy similar al promedio reportado por Nicholson. Es de llamar la atención que en pacientes que son capaces de sostener parte de su trabajo respiratorio, como es el caso del SHC el tiempo de adaptación sea mayor. Probablemente esta diferencia está relacionada con la edad.

Se ha reportado que los pacientes con MD tienen concurrentemente obstrucción de vía aérea superior, por lo que con el retiro de la cánula de traqueostomía pueden presentar complicaciones como apnea obstructiva del sueño ${ }^{13}$. Diep revisó 15 pacientes con SHC congénito y traqueostomía, encontrando que posterior a la colocación del marcapasos 11 pacientes fueron decanulados exitosamente, en promedio a los 12,2 meses $(0,6-40,6)^{14}$. En nuestro caso a 24 meses de seguimiento posterior a la cirugía aún no se retira cánula de traqueostomía. Dentro de los factores para no haberlo realizado están la presencia de retraso en el desarrollo psicomotor y alteración de la mecánica de la deglución.

\section{Conclusiones}

El presente reporte y la revisión de la literatura sugieren que la implantación del MD es una opción factible, potencialmente segura y costo efectiva para disminuir o eliminar la dependencia a la ventilación mecánica y mejorar la calidad de vida en pacientes con SHC adquirido.

\section{Responsabilidades Éticas}

Protección de personas y animales: Los autores declaran que los procedimientos seguidos se conformaron a las normas éticas del comité de experimentación humana responsable y de acuerdo con la Asociación Médica Mundial y la Declaración de Helsinki.

Confidencialidad de los datos: Los autores declaran que han seguido los protocolos de su centro de trabajo sobre la publicación de datos de pacientes.

Derecho a la privacidad y consentimiento informado: Los autores han obtenido el consentimiento informado de los pacientes y/o sujetos referidos en el 
artículo. Este documento obra en poder del autor de correspondencia.

\section{Conflicto de intereses}

Los autores declaran no tener conflicto de intereses.

\section{Agradecimientos}

Agradecemos al Dr. Fernando Bracho por su gestión para conseguir el financiamiento de la compra del dispositivo.

\section{Referencias}

1. Ducko CT. Clinical advances in diaphragm pacing. Innovations (Phila). 2011;6(5):289-97.

2. Sieg EP, Payne RA, Hazard S, Rizk E. Evaluating the evidence: is phrenic nerve stimulation a safe and effective tool for decreasing ventilator dependence in patients with high cervical spinal cord injuries and central hypoventilation? Childs Nerv Syst. 2016;32(6):1033-8.

3. Ali A, Flageole H. Diaphragmatic pacing for the treatment of congenital central alveolar hypoventilation syndrome. J Pediatr Surg. 2008;43(5):792-6.

4. Romero-Ganuza FJ, GambarruttaMalfatti C, Diez de la Lastra-Buigues E, et al. Diaphragmatic pacemaker as an alternative to mechanical ventilation in patients with cervical spinal injury. Med Intensiva. 2011;35(1):13-21.

5. Nicholson KJ, Nosanov LB, Bowen KA, et al. Thoracoscopic placement of phrenic nerve pacers for diaphragm pacing in congenital central hypoventilation syndrome. J Pediatr Surg. 2015;50(1):7881.

6. Abdunnur SV, Kim DH. Phrenic Nerve Stimulation: Technology and Clinical Applications. Prog Neurol Surg. 2016;29:64-75.

7. Le Pimpec-Barthes F, Legras A, Arame A, et al. Diaphragm pacing: the state of the art. J Thorac Dis. 2016 Apr;8(Suppl 4):S376-86.

8. Khong P, Lazzaro A, Mobbs R. Phrenic nerve stimulation: the Australian experience. J Clin Neurosci. 2010;17(2):205-8.

9. Shaul DB, McComb JG, Keens TG. Thoracoscopic placement of phrenic nerve electrodes for diaphragm pacing. Pediatr Endosurg Innov Tech. 1998;2:1015.

10. Shaul DB, Danielson PD, McComb JG, Keens TG Thoracoscopic placement of phrenic nerve electrodes for diaphragmatic pacing in children. J Pediatr Surg. 2002;37(7):974-8.

11. Romero FJ, Gambarrutta C, GarciaForcada A, et al. Long-term evaluation of phrenic nerve pacing for respiratory failure due to high cervical spinal cord injury. Spinal Cord. 2012;50(12):895-8.

12. Hirschfeld S, Exner G, Luukkaala T, Baer GA. Mechanical ventilation or phrenic nerve stimulation for treatment of spinal cord injury induced respiratory insufficiency. Spinal Cord. 2008;46(11):738-42.

13. Wang A, Kun S, Diep B, et al. Obstructive sleep apnea in patients with congenital central hypoventilation syndrome ventilated by diaphragm pacing without tracheostomy. J Clin Sleep Med. 2018;14(2):261-4.

14. Diep B, Wang A, Kun S, et al. Diaphragm pacing without tracheostomy in congenital central hypoventilation syndrome patients. Respiration. 2015;89(6):534-8 